\title{
Isolation and Characterization of Melanin Pigment from Bacillus subtilis ${ }^{\dagger}$
}

\author{
Deepa G. Muricken ${ }^{1, *}$, Raihana Nesreen ${ }^{1}$, Sahala A.M. ${ }^{1}$, Shajla ${ }^{1}$, Soya K. Sunny ${ }^{1}$, Krishnapriya C.S. ${ }^{1}$ \\ 1 Department of Biochemistry, St. Mary’s College Thrissur, Kerala \\ * Correspondence: murickendeepa@gmail.com; \\ $\dagger$ Presented at International e-Conference on Bioengineering for Health and Environment (ICBHE 2020)
}

Received: 5.07.2020; Revised: 10.07.2020; Accepted: 12.07.2020; Published: 15.07.2020

\begin{abstract}
Melanin is nearly a ubiquitous pigment synthesized by living organisms in the course of hydroxylation and polymerization. Melanin has immense application potential in the field of agriculture, cosmetics, and pharmaceutical industries. The aim of this study was to obtain the melanin pigment produced by Bacillus subtilis using $\mathrm{T}$ medium and study the biological and chemical characteristics of the pigment. Melanin pigment production in Bacillus was analyzed and was optimized at different temperatures and $\mathrm{pH}$ for optimal production. The pigment was confirmed by its chemical characterization. The melanin pigment obtained was water-soluble and was confirmed to be photoprotective using Ultraviolet-Visible spectrum analysis, which showed maximum absorption in the UV region (200-300 nm), but diminished towards the visible regions. The pigment also showed antioxidant activity. Fourier Transformation Infrared spectroscopy analysis confirmed the crude melanin extract obtained as melanin. DNA binding property of melanin was studied. UV- Visible spectroscopic methods shows that melanin is able to bind DNA and impact protection. The pigment was analyzed for its application in the field of agriculture. It had shown to impart UV protection to UV exposed seeds during germination. Melanin also found to enhance the growth of plants when studied under laboratory conditions.
\end{abstract}

Keywords: Melanin; Bacillus subtilis; FTIR; UV protection; Plant growth promotion.

(C) 2020 by the authors. This article is an open-access article distributed under the terms and conditions of the Creative Commons Attribution (CC BY) license (https://creativecommons.org/licenses/by/4.0/).

\section{Funding}

This research received no external funding.

\section{Acknowledgments}

This research has no acknowledgment.

\section{Conflicts of Interest}

The authors declare no conflict of interest. 